\title{
A simple method of projecting Mach bands, color mixtures, and variable contrast sinusoidal gratings
}

\author{
S. M. ANSTIS \\ University of Bristol, 10 Berkeley Square, Bristol BS8 1HH, England \\ and \\ J.P. COMERFORD \\ York University, 4700 Keele Street, Downsview, Ontario, Canada
}

\begin{abstract}
This paper describes a simple method of projecting Mach bands, color mixtures, and variable-contrast sinusoidal gratings.
\end{abstract}

The standard method of displaying a grating with a sinusoidal luminance profile is on an oscilloscope screen (Campbell \& Green, 1965; Schade, 1956). This method is useful and versatile, but the luminance is usually well below $100 \mathrm{~cd} / \mathrm{m}^{2}$ because the tube phosphor is apt to burn out and the size of the display is limited to the size of the tube face. It is possible to project an enlarged image of the tube face onto a ground-glass back-projection screen, using the projection lens from a slide projector, but this further reduces the available luminance. Display oscilloscopes with 19-in. screens are available 1 but basically these are modified television monitors with magnetic deflection coils. Magnetic deflection has an inherently lower bandwidth than the electrostatic deflection used on ordinary small-screen oscilloscopes, and this can lead to flicker problems. Also they are not cheap.

Large bright gratings of about $250 \mathrm{~cd} / \mathrm{m}^{2}$ can be projected onto a screen for classroom display very cheaply, using an ordinary overhead projector. A sinusoidal curve whose amplitude and wavelength are both $2.5 \mathrm{~cm}$ is drawn down the middle of a piece of black paper with the aid of a Berol "Rapidesign" No. 305 sine-curve stencil (Berol Corporation, Burbank, California) or the similar Standardgraph No. 3330 stencil (Standardgraph, Filler \& Ficbig, Germany). The paper is cut in half along this wavy line with a razor knife, giving two sinusoidal edges. These are laid on the horizontal bed of an American Optical Apollo Six overhead projector with the black peaks touching each other so that the whole bed surface is blacked off except for an hourglass-shaped slit whose width is sinusoidally modulated, ranging from zero where the peaks touch to $5 \mathrm{~cm}$ where the troughs are opposite each other (Figure 2a). When the projector lamp is switched on, an enlarged sharply focused image of the horizontal

S. M. Anstis was supported by Grant B/SR/48368 from the Science Research Council.
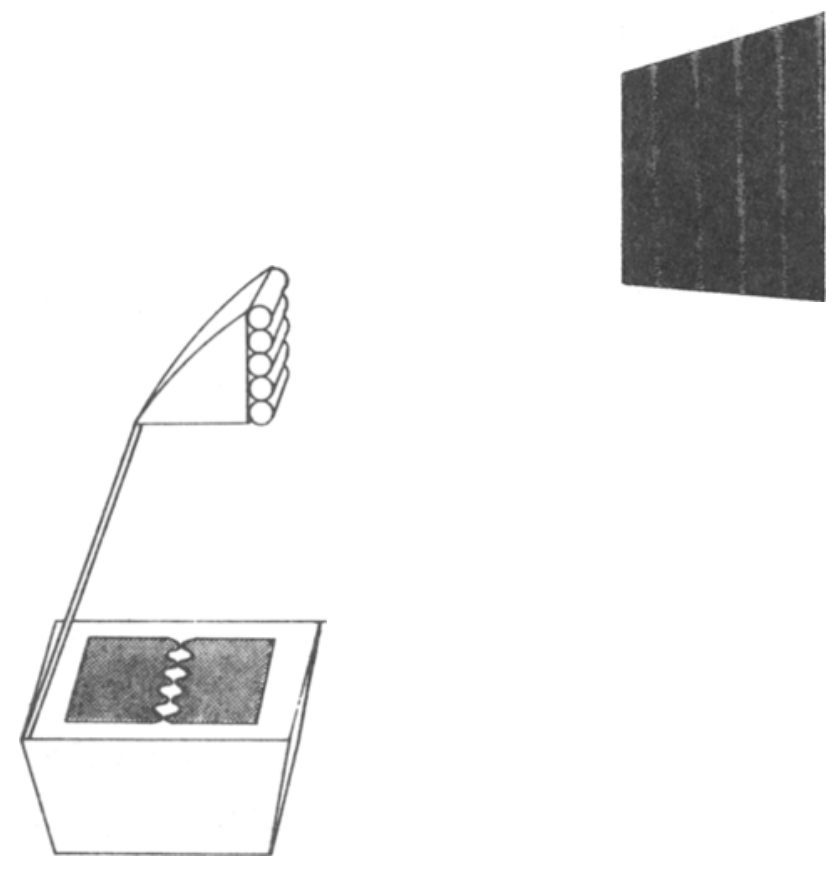

Figure 1. Projection of sine-wave gratings on a screen. Opaque masks for the wave forms were placed over the light source on the projector bed. The Plexiglas astigmatizing rods can be fitted inside the projection-lens housing, or outside, as shown.

wavy-edged slit is back-projected on a ground-glass screen. To convert the variations in width of this bright slit into parallel vertical bars of varying brightness on the screen, the projected image is vertically stretched out or smeared by an astigmatic cylindrical lens. This lens is made by mounting parallel horizontal Plexiglas rods, $1 \mathrm{~cm}$ in diam, to a Plexiglas plate. The rods have to touch all along their lengths when mounted in place, since even the slightest clear slit left between adjacent rods allows an unsmeared image of the wavy slit to appear on the screen. On the projector we use, this 

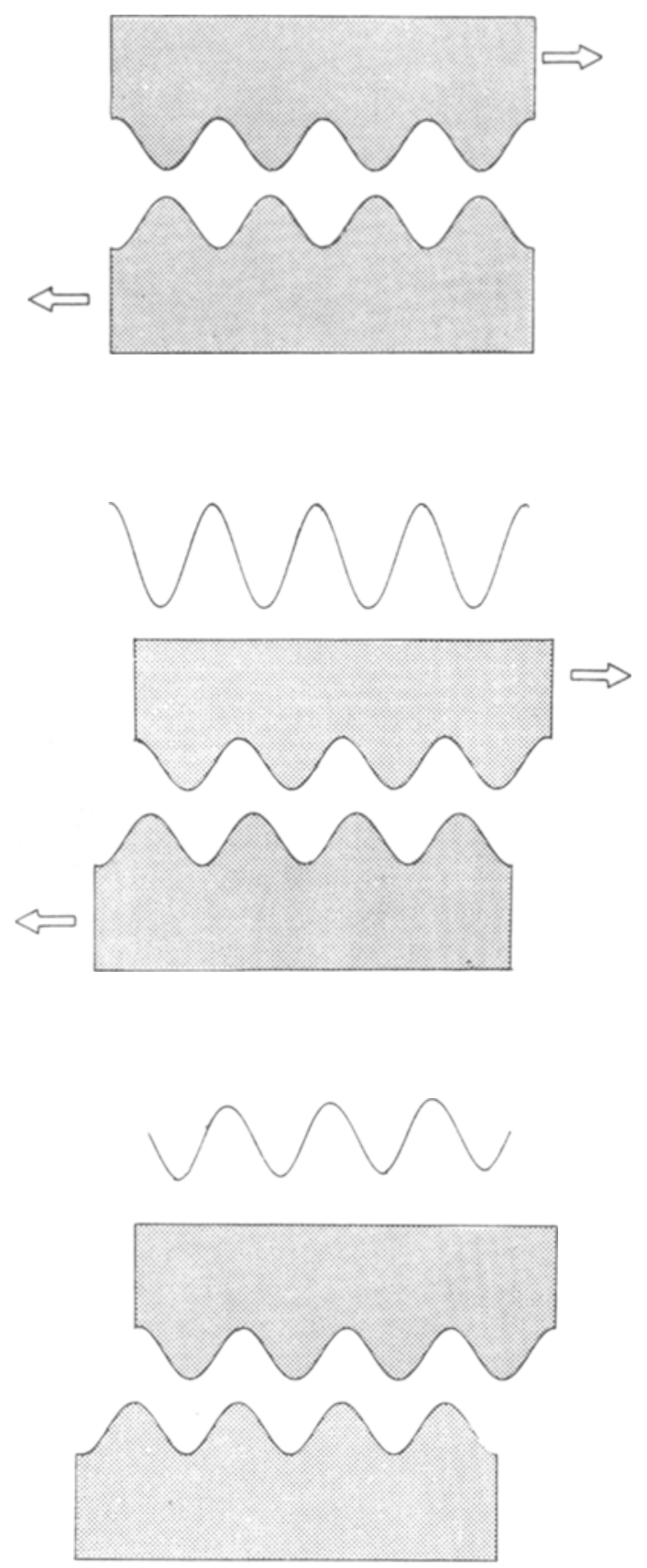

Figure 2. Variable-contrast gratings obtained by shifting masks of the same frequency sine wave relative to each other: (a) when peaks are opposite each other, masks are in phase and contrast (bottom line) is maximum; (b) contrast falls when masks are shifted in opposite directions; and (c) approaches zero when masks are in antiphase, with peaks of one mask lined up with troughs of other mask, such that the slit is of equal width at all points.
Plexiglas device fits snugly inside the projection-lens housing, which can be hinged open to allow its insertion between the front and rear lens elements. On projectors without this facility, the device can be placed just in front of the projection lens, as shown in Figure 1, but the results are not quite so satisfactory. Alternatively, a sheet of horizontally ribbed or beaded Plexiglas (Edmund Scientific Corporation lenticular screen No. 80130 costing about $\$ 3.00$ ) is almost equally effective and much more labor saving.

Calibration using a Spectra Prichard photometer verifies that with the astigmatic cylindrical lens in place there is a linear relationship (plus or minus $4 \%$ ) between the size of an unmasked gap on the projector bed and the amount of light projected on the screen. In particular, a gap formed by two masks with sine-wave-shaped edges, as in Figure $2 a$, projects a sine-wave distribution of light on the screen.

To keep the bars in the grating reasonably narrow, the screen is placed $1 \mathrm{~m}$ in front of the projector, which is as close as the focusing arrangments allows. This gives a magnification factor of three, with a grating wavelength of $7.5 \mathrm{~cm}$ and, hence, a spatial frequency of about 2 cycles/deg at a viewing distance of $10 \mathrm{~m}$. It is not easy to obtain higher spatial frequencies by cutting out smaller stimulus-sine-wave curves, since any cutting errors are magnified. One solution might be to cut out large sine curves and reduce them photographically.

This method resembles the color wheel, whose rapid rotation mixes black and white areas on the spinning disk to give shades of grey proportional to the black and white areas. But this method produces a larger, brighter picture and is much more flexible because it separates the mixture or fusion achieved by the cylindrical rods from the stimulus patterns on the projector bed. In this way, the projected luminance profiles can be altered in real time by pushing pieces of paper around, with no need to start and stop rotations. Békésy $(1960 ; 1967$, p. 73) described a method of producing Mach bands by looking at a slit of variable width through a rotating glass prism of square cross section, which transformed the geometrical pattern of the opening into a pattern of light density, as our method does. Békésy's technique shows his usual elegance, but our method has the advantage of needing no moving parts and it can be viewed by a large group of observers.

The contrast of the grating is varied by exploiting the fact that the sum of two sine waves of the same frequency is always another sine wave, whose amplitude depends upon the phase relation of its two component sine waves. To reduce the amplitude (contrast) of the grating, one of the two pieces of wavy-edged paper is moved slowly past the other in a direction parallel to the slit axis (Figure $2 b, c$ ). As the wavy edges move gradually out of phase, the shape of the slit changes from an hourglass to a wavy river, and the grating contrast falls steadily. When the edges are in antiphase, they are still 

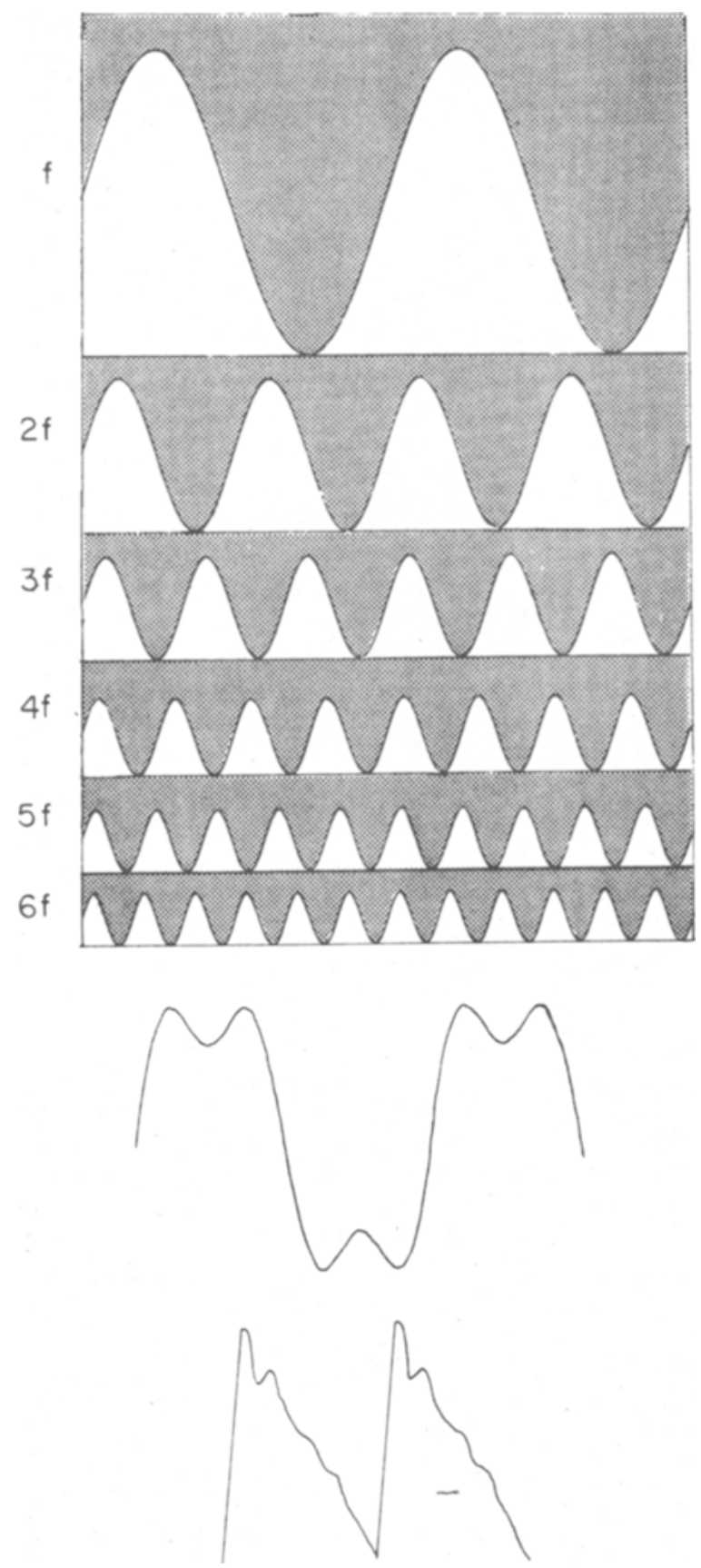

Figure 3. Fourier synthesis of square and sawtooth waves. The mask is composed of sine-wave cutouts with frequencies $\mathrm{f}, \mathbf{2 f}, \mathbf{3 f}$, $4 f, 5 f$, and $6 f$, with relative amplitudes of $1,1 / 2,1 / 3,1 / 4,1 / 5$, and $1 / 6$, respectively. When all but the fundamental and the third and fifth harmonics (f, 3f, 5f) are covered, a grating is projected whose luminance profile approximates a square wave (b). Projecting all the wave forms together produces a luminance profile approximating a sawtooth (c).

wavy but parallel, so the width of the wavy slit is the same at all points, the smeared field of light on the screen is uniform, and the grating's contrast is (ideally) zero. Fine control over contrast can be obtained by the

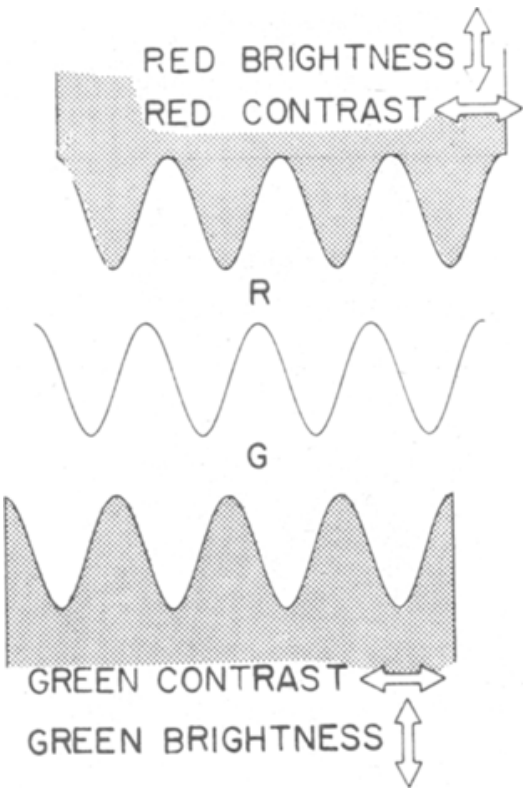

Figure 4. Colored gratings. $A$ red and a green filter are cut with a snugly fitting common sinusoidal edge and taped to be stationary on the projector bed. Black paper masks are laid on top of these filters. Varying the position of the masks varies the contribution of the two filters to the overall luminance and contrast of the projected grating. Horizontal shifts of the masks, as in Figure 2, alter the contrasts, and vertical shifts alter the luminances.

use of low-amplitude wavy edges. This might be useful in setting gratings to threshold.

If the wavy edges move in opposite directions through several cycles, a stationary grating can be seen on the screen whose contrast waves and wanes repetitively, reversing in contrast with the dark bars becoming light and vice versa. A physicist would call this a standing wave, psychophysicists call it counterphase flicker.

To increase the mean luminance of the grating, the slit is simply made wider. This adds a dc veiling luminance to the ac grating and, of course, reduces proportionately the depth of modulation. To alter the mean luminance without altering the depth of modulation, neutral-density filters can be moved in and out of the optical path.

Gratings can be added together optically by placing two or more sine-wave slits of different spatial frequencies on the projector bed with their axes parallel. Principles of Fourier synthesis of sawtooth and square waves can be demonstrated with the aid of Figure 3 which contains sine-wave cutouts of frequencies $f, 2 f, 3 f$, $4 f, 5 f$, and $6 f$, with relative amplitudes of $1,1 / 2,1 / 3$, $1 / 4,1 / 5$, and $1 / 6$, respectively. These can all be drawn from the "Rapidesign" stencil. First, the pattern is laid on the projector bed with only the first, third, and fifth harmonics exposed: the even-number harmonics are overlaid by the horizontal strips of an E-shaped cutout. A sheet of opaque paper is gradually withdrawn downwards to uncover the first, third, and fifth 

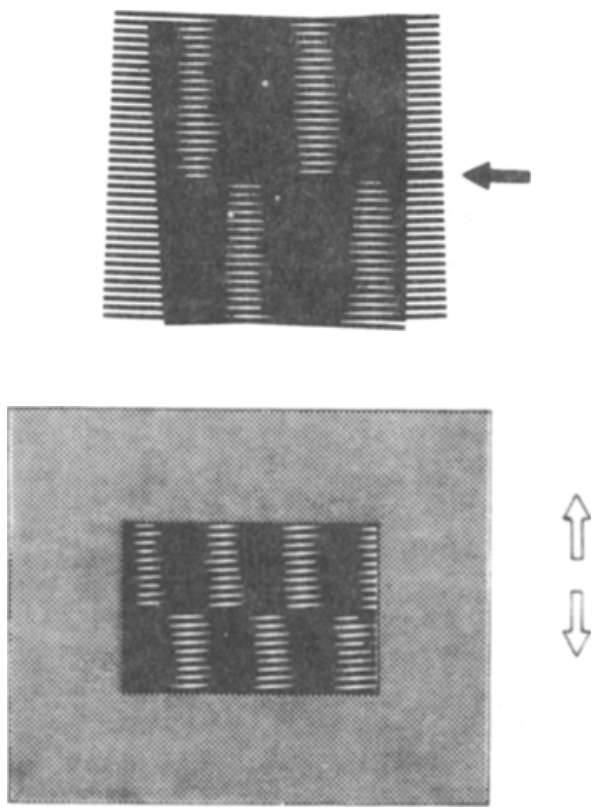

Figure 5. Gratings of variable spatial frequency and contrast using moiré fringes. Spatial frequency of fringes are varied by small rotations of one striped pattern relative to the other (not illustrated). To vary the contrast, one striped pattern is first cut and overlapped with itself to reverse the spatial phase. This produces spatial-phase reversal in the resulting moire fringes (arrowed in a). A rectangular aperture (b) can be placed over the stationary fringes to select a ratio of in-phase to antiphase moiré fringes. When the ratio is $1: 1$ as shown, contrast of the resulting grating approaches zero. Shifting aperture up or down increases the contrast.

harmonics in turn so that these add optically on the screen to build up a grating whose luminance profile approximates a square wave. The E-shaped cutout is then slowly pulled off sideways, exposing the second, fourth, and sixth harmonics, which transforms the square-wave grating on the screen into an approximate sawtoothed grating.

Colored gratings can be cut out of colored gelatines and black paper (Figure 4). A red-green grating is composed of a red grating superimposed on an antiphase green grating. Figure 4 shows that the luminance and the contrast of the red and green component gratings can each be adjusted independently by moving the black puper around. The green gelatine can be replaced by grey gelatine of equal density in order to produce a red grating whose luminance, contrast, and saturation can be varied independently.

Gratings of variable spatial frequency are projected by replacing the wavy slit with a moire fringe pattern. Two identical transparencies of fine repetitive stripes are superimposed at more or less horizontal orientations which differ by $5.20 \mathrm{deg}$. The horizontal stripes are blurred astigmatically, but they produce coarse vertical moiré fringes that are projected on the screen as a vertical grating whose spatial frequency can be varied by small alterations in the relative orientations of the two transparencies. The striped patterns consist of pieces of
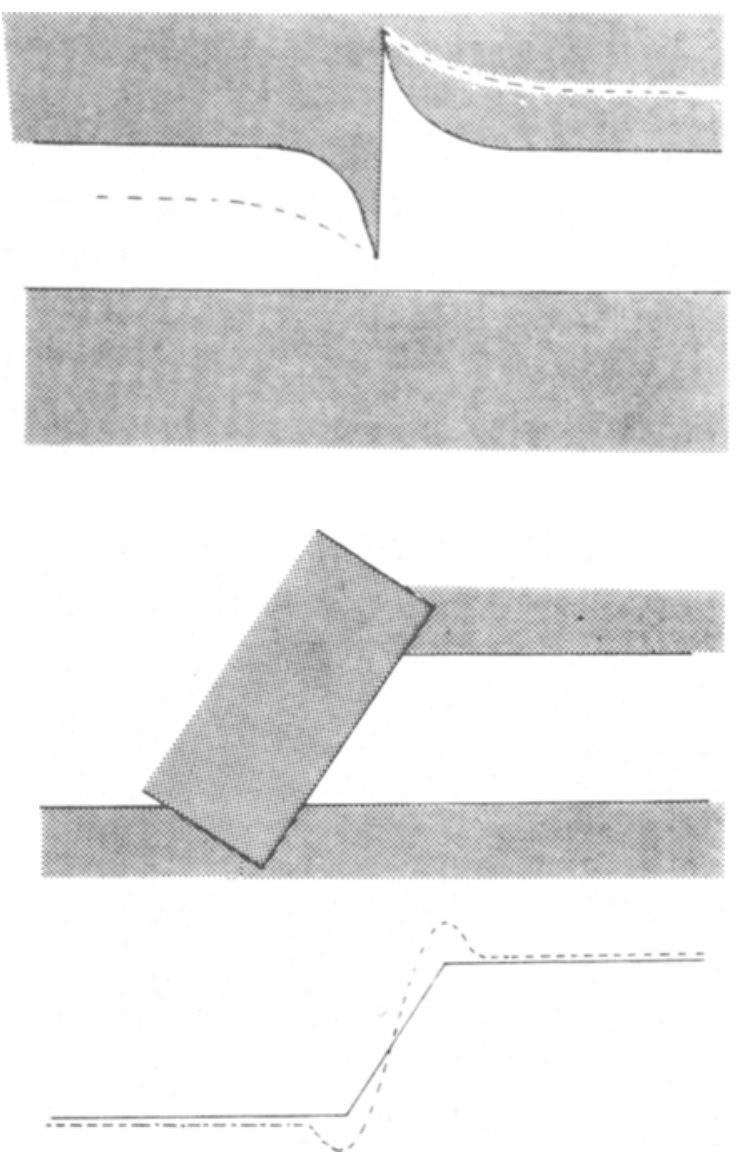

Figure 6..a. The Craik-O'Brien-Cornsweet illusion. A slit cut to give the luminance profile shown as the solid line gave a sensation like the dotted line, with the left half apparently much darker than the right. b. Mach bands. An opaque card laid at an angle actoss the broad slit gave a luminance profile shown as the solid line in c, but a sensation like the dotted line.

Letraset "Letratone" LT108, removed from their protective backing and attached to transparent acetate sheet or cel. Finer Letratone stripes such as LT 70 give higher spatial frequencies but do not produce as good a contrast on the screen. "Zip-a-Tone" striped patterns (Para-Tone, Inc.) can be used also.

The contrast of the moire fringe grating can be varied by exploiting the fact that the sum of a pair of antiphase sine waves of the same frequency is always another sine wave, whose amplitude depends upon the relative amplitude of its two component sine waves. One of the finely striped "Letratone" patterns is cut in half along a stripe and overlapped by one stripe's width so that the two halves of the pattern are in opposite spatial phase (Figure 5a). Thus, when the second striped pattern is superimposed, the resulting moiré fringes are also reversed in spatial phase over half of the field. A rectangular aperture cut in black paper overlays the moiré fringes, and its position selects the ratio of in-phase to antiphase fringes that are projected and 
optically added on the screen (Figure 5b). When only one set of fringes is exposed through the aperture, the grating contrast is maximum. As antiphase fringes are added in, the grating contrast falls steadily, becoming (ideally) zero when the areas of in-phase and antiphase fringes are equal. Rapid up-and-down movements of the aperture over the stationary fringes produce counterphase ficker on the screen. Note that this method holds the space-averaged luminance constant.

Reverting to the slit method, Mach bands can be projected very readily. The effect of step and ramp profiles can be explored with $3 \times 5$ in. file cards that are moved around on the projector bed. The Craik-O'Brien-Cornsweet illusion (Comsweet, 1970; Figure 11.2) gave a very strong impression that the projected area on the left in Figure 6a was darker than that on the right. The effect was judged to be far more striking than from an ordinary spinning color wheel. Ratliff (1965) and Békésy (1967) contain many suggestions for Mach band luminance profiles. These can be quickly cut out of black paper and projected. The setup can be used also as a colorimeter to demonstrate color-mixture effects qualitatively. In particular, the Young-Helmholt $z$ three-color theory can be dramatized by cutting out of black paper the well-known spectral response curves for color matching using red, green, and blue primaries (Figure 7). Sinusoidal curves from the stencil are used as an approximation to the true color-matching curves, and the blue curve is arbitrarily made $.65 \%$ taller than the others. The holes cut in the paper are covered with blue (No.98), green (No.61), and red (No. 29) Wratten filters. When projected, the resulting color mixture looks like a physical spectrum.

\section{REFERENCES}

Békésy, G. von. Neural inhibitory units of the eye and skin: Quantitative description of contrast phenomena. Journal of the Optical Society of America, 1960,50, 1060-1070.

Békésy, G. von. Sensory inhibition. Princeton University Press. 1967

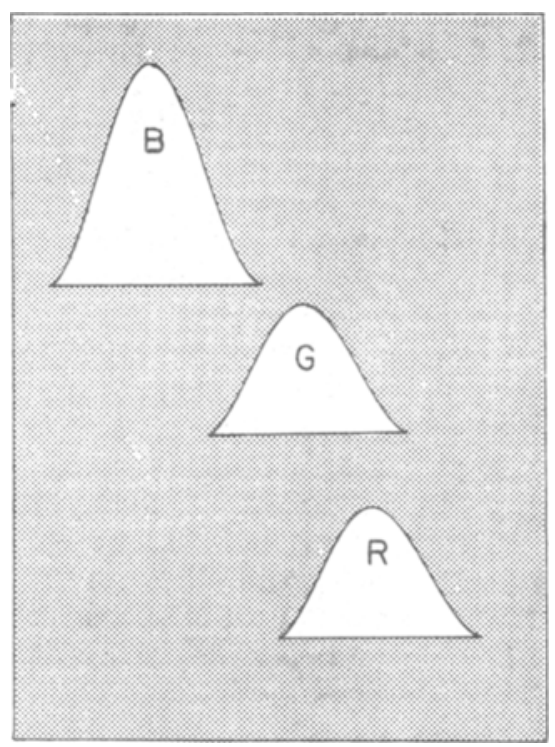

Figure 7. Color mixture. Color-matching functions described the proportions of blue, green, and red primaries needed to match test hues across the physical spectrum. Curves approximating these functions are cut out of black paper and overlaid with blue (No. 98), green (No. 61), and red (No. 29) Wratten filters. When astigmatised vertically, this produces a "spectrum" of colors ranging from blue through green and yellow to red.

Campbell, F. W. \& Green, D. G. Optical and retinal factors affecting visual resolution. Journal of Physiology, London, $1965,181,576-593$

Cornsweet, T. N. Visual perception 1970.

Ratliff, F. Mach bands. Quantitative studies on neural networks in the retina. San Francisco: Holden-Day, 1965.

Schade, O. H. Optical and photoelectric analog of the eye. Journal of the Optical Society of America, 1956, 46, 721-739.

\section{NOTE}

1. For instance, the Lan-Scope from Lan Electronics, Slough, Bucks, England.

(Received for publication September 20, 1974; revision received November $12,1974$. ) 